www. revistadyo.com

\title{
Aplicación de técnicas Lean Construction a través de un método de Action Research en los procesos de gestión de una empresa constructora
}

Fernández Vázquez-Noguerol, M., Rodríguez-García, M. y Prado-Prado, J. C.

Recibido: 6 de Marzo de 2018 / Aceptado: 17 de Mayo de 2018

\section{Resumen}

En este artículo, se desarrolla un método de Action Research para implementar técnicas de Lean Construction en los procesos de gestión de una empresa constructora. A través de la creación de equipos formados por profesionales de distintas áreas, se busca definir e implementar diferentes mejoras. Nuestra investigación se divide en tres fases: captación de datos, definición de las líneas de actuación e implementación y seguimiento del plan de actuación. Los resultados de nuestro estudio sugieren que la colaboración entre los diferentes departamentos ayuda a lograr un cambio empresarial significativo y a potenciar la implementación de la filosofía Lean Construction.

\section{Palabras clave}

Lean Construction, Action Research, Mejora de Procesos, Equipos Multidisciplinares.

\section{Introducción}

El término Action Research tiene una historia compleja porque no es una disciplina académica única, sino un enfoque de investigación que ha surgido a lo largo del tiempo en diversos campos de aprendizaje (Brydon-Miller at al., 2003). Esta metodología está basada en principios de aprendizaje experimental, siguiendo un método de estudio de caso con el objetivo de conocer y definir los procesos organizativos complejos de las empresas (Yin, 2014). El estudio de caso juega un papel fundamental en este tipo de investigaciones para ayudar en la resolución de problemas, ya que proporciona rigor académico y relevancia práctica a los investigadores (McManners, 2016).

En la actualidad, la metodología de Action Research continúa siendo una herramienta muy utilizada en las diferentes ramas de conocimiento (Farooq y O'Brien, 2015; Kaipia et al., 2017; McManners, 2016; Rydenfält et al., 2017). Las primeras etapas del proceso de Action Research son la definición de la problemática encontrada y la exploración reflexiva de las dificultades, por ello la participación de los profesionales a través de equipos de trabajo es clave en este tipo de estudios. En general, los equipos de trabajo suelen ser car-

\footnotetext{
Fernández Vázquez-Noguerol , M.

marfernandezvazquez@uvigo.es

Rodríguez-García, M.

miguelgarcia@uvigo.es

Prado-Prado , J. C.

jcprado@uvigo.es

Grupo de Ingeniería de Organización. Dpto. de Organización de Empresas y Marketing. Escuela de Ingeniería Industrial. Universidad de Vigo. c/Maxwell, s/n - 36310 Vigo, España
}

acterizados por un alto compromiso y una gran participación (McGrath, 2001). En consecuencia, la aplicación de Action Research representa una secuencia de eventos y enfoques utilizados para resolver problemas, a la vez que se adquiere conocimiento del proceso de cambio (Gutierrez et al., 2015). En este estudio se trabajó con diferentes equipos de trabajo siguiendo la metodología de krAft, comúnmente utilizada en este tipo de análisis y basada en el concepto del aprendizaje de Kolb (1984) (Sunding y Odenrick, 2010). La metodología krAft está centrada en la importancia del aprendizaje experimental y en facilitar un fructífero encuentro entre las perspectivas de los diferentes participantes del equipo. Por esta razón, el modelo aquí presentado está principalmente ligado a asegurar una acción reflexiva de la estructura interna de la organización, junto a la entrada de personas externas (investigadores), que facilitan la coordinación y seguimiento de las mejoras propuestas.

Por otro lado, la correcta gestión de una obra en el sector de la construcción ha sido un desafío a lo largo de la historia y, aún hoy en día, lo sigue siendo (Dave et al., 2016). Si bien la especialización de tareas benefició a muchas industrias después de la revolución industrial, la industria de la construcción avanzó aún más hacia la fragmentación de los roles (Kumaraswamy, 1999). En la actualidad, se afirma cada vez más que esta evolución fue muy importante, perjudicando el trabajo en equipo y contribuyendo significativamente a sobrecostes inaceptables y plazos excesivos. En consecuencia, la construcción ha sido criticada durante mucho tiempo por su baja productividad y por los problemas relacionados con la gestión de los procesos en la ejecución de las obras (Kagioglou et al., 2000; Teicholz et al., 2001). Un análisis realizado por Horman y Kenley (2005) mostraba que, durante los últimos treinta años, casi el cincuenta por ciento del tiempo dedicado a actividades en la construcción no aportaba valor. 
En este ámbito, Koskela (1992) definió las bases para aplicar la producción sin pérdidas al sector de la construcción, lo que pasó a denominarse Lean Construction. Esta técnica está centrada en la reducción de desperdicios, en aumentar el valor para el cliente y en la mejora continua (Dave et al., 2016). La transferencia de esta metodología Lean desde la producción industrial a la industria de la construcción todavía se considera crítica, especialmente en la práctica, debido a que la construcción se focaliza en la producción de un único elemento (Berner et al., 2016). Así, autores como Azambuja y O'Brien (2009), se han centrado en proporcionar las principales diferencias entre las cadenas de suministro de la fabricación y de la construcción, con el objetivo de mejorar la eficiencia y reducir los costes.

Este estudio muestra la suma importancia que presenta la mejora de los procesos en el sector de la construcción a través de la Action Research, una metodología muy poco utilizada hasta la fecha en estudios sobre este sector. De igual modo, Dave (2017) concluye que la efectividad de las técnicas de mejora de procesos en la construcción aún no se ha analizado con detalle. Además, cabe destacar que hay una escasez de estudios de investigación de la aplicación del Lean Construction dentro del sector de la construcción (Tezel et al., 2017). Todo esto, unido a la falta de investigación experimental para demostrar cómo las organizaciones difunden y promulgan los principios del pensamiento Lean para lograr las recompensas prometidas (Sarhan y Fox, 2012), originan el desarrollo de este estudio.

Por otra parte, en este sector es frecuente el uso de otra técnica que tiene ciertas similitudes con la Action Research a la hora de analizar la problemática observada. Se conoce como Design Science y difiere principalmente de la Action Research en que la primera busca desarrollar conocimientos genéricos que se puedan transferir a diversos contextos dentro de un mismo dominio, mientras que la segunda apunta a mejoras específicas de casos concretos (van Aken at al., 2016). Como ejemplo de uso, autores como Dave et al. (2016) utilizan las primeras fases de Design Science para analizar las problemáticas presentes en la gestión de la construcción. Otros autores tratan de utilizar de manera conjunta el método de ciencia del diseño junto a la Action Research para realizar diferentes desarrollos (Hjalmarsson et al., 2010; Choma et al., 2015; Haj-Bolouri et al., 2016).

El objetivo de nuestro estudio es definir y desarrollar un modelo de Action Research para facilitar el desarrollo organizacional en el sector de la construcción, basándose en el uso de herramientas de Lean Construction. Se trata de un único estudio de caso enfocado a la mejora de los procesos de gestión. La empresa presenta tres grandes problemas que son trasversales a toda la organización. Por un lado, el ratio de obtención de proyectos respecto al número de ofertas elaboradas es muy bajo. Por otro, es frecuente que las obras que se gestionan conlleven a importantes sobrecostes y que, además, se terminen fuera de plazo. Mediante la realización del estudio se persigue fomentar la colaboración entre los profesionales, desarrollar propuestas de mejoras y localizar research findings que puedan ser de utilidad para la investigación. En este tipo de estudios el grado de aprendizaje depende de diversos factores entre los que cabe destacar, la elección del caso a observar, la preparación de la observación en función del objetivo buscado, la forma de realizar la misma acción y por último la reflexión realizada a partir de la propia experimentación.

El documento está estructurado de la siguiente manera: la Sección 2 presenta una descripción detallada de la metodología de investigación, en donde se describen las 3 fases del estudio. Una fase inicial de captación de datos, en la cual se presentan los problemas observados y su relación con la literatura actual sobre el sector de la construcción, especialmente en lo relativo a las técnicas de Lean Construction. Una segunda fase de análisis y definición de las líneas de actuación donde se definen los equipos de trabajo. Y una tercera fase donde se explica el proceso de implementación y seguimiento de las líneas de actuación en cada uno de los equipos. Posteriormente, la Sección 3 desarrolla los resultados de cada una de las fases descritas. En el último apartado, Sección 4, se presentan las conclusiones del estudio y las pautas para futuras investigaciones.

\section{Metodología}

Durante el estudio, los investigadores necesitaron de la colaboración plena del personal de la empresa para ser capaces de encontrar las oportunidades de mejora claves que ayudasen a solucionar los problemas que se presentaron. Por ello, el diseño del estudio se consideró Action Research, debido a que los profesionales trabajaron, junto con un grupo de investigadores, en la búsqueda de un objetivo común. La filosofía de Action Research permite involucrar al investigador con las partes interesadas como un participante activo objetivo dentro del caso de estudio (McManners, 2016). Por parte del grupo de investigadores, estos son considerados expertos en proyectos industriales con la participación del personal que nacen en base a una problemática presente en una empresa. Para solventar la problemática objeto de estudio, el grupo de investigadores analizó la situación y definió un plan de actuación tanto a corto como a medio y largo plazo. El proceso de investigación se dividió en tres fases (Figura 1). 
Figura 1 Ciclo de Action Research adaptado de Coughlan y Coughlan (2002) y Fagundes et al. (2017).

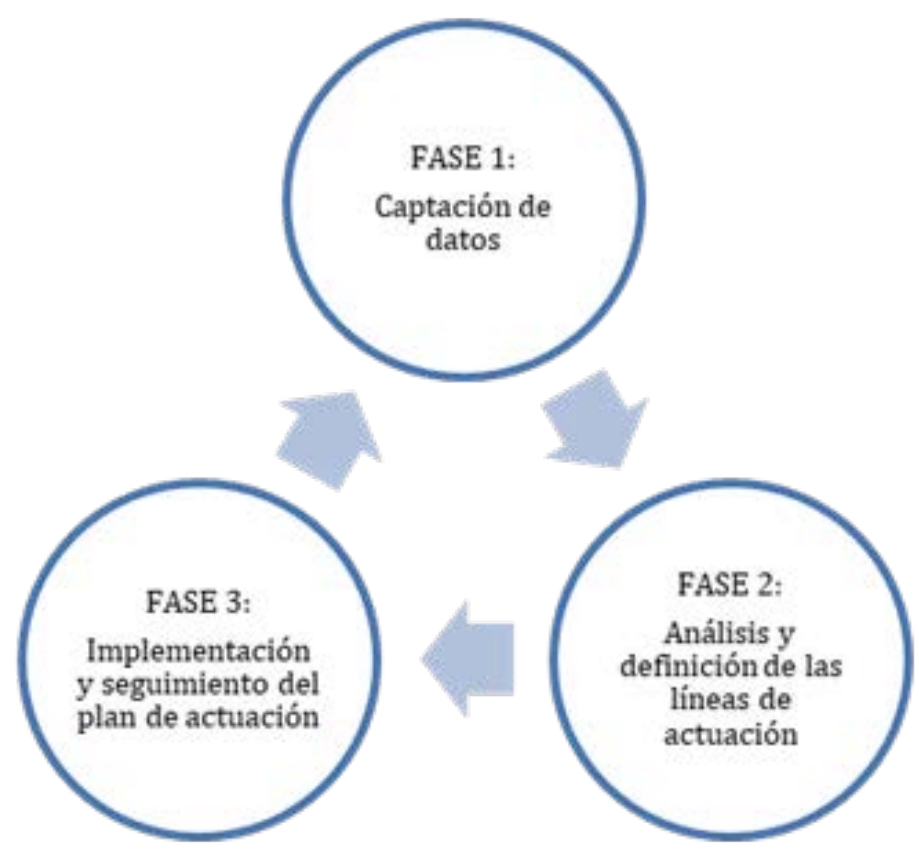

La Fase 1 es una fase preparatoria de conocimiento para averiguar cuáles son los problemas más importantes a abordar en la empresa. En esta etapa se expone la situación de la misma a través del conocimiento interno de los profesionales y se complementa con la visión externa de los investigadores. Así, se van relacionando los problemas observados en el caso específico con los presentados en la literatura actual. En esta fase la orientación del equipo hacia el objetivo definido inicialmente tiene que ser completa. En nuestro caso, fueron las tres problemáticas principales (bajo ratio de conversión oferta/pedido, sobrecostes y retrasos en las obras) las que guiaron el proceso de extracción de información de los entrevistados por parte de los investigadores.

Se realizan, por tanto, entrevistas tanto individuales como departamentales con objeto de conocer los diferentes problemas existentes complementarios al problema inicial. Al mismo tiempo, se deja definido el rol de los investigadores a lo largo del estudio y el alcance de su actuación (van Aken et al., 2016). Los investigadores son los encargados de ir recopilando la información de las entrevistas, correspondiendo a uno de ellos la labor de ir transcribiendo cada una de las mismas. A la vez, muestran su visión permitiendo complementar el enfoque interno y orientando la información hacia la búsqueda de los objetivos definidos. Con motivo de estructurar adecuadamente esa información y tras realizar las primeras entrevistas con los diferentes departamentos, los investigadores junto a los responsables de los departamentos, identifican las prioridades de mejora utilizando una herramienta de mapeo de procesos. Esta técnica de análisis de procesos permite conocer en detalle un proceso ya implementado sobre el cual se desea aplicar ciertas mejoras. Cabe destacar la importancia de desarrollar estos mapas de proceso debido a que las organizaciones deben medir y mejorar constantemente sus procesos para seguir compitiendo en el mundo empresarial dinámico actual (Paim et al., 2008).

La Fase 2 consiste en la aplicación de la reingeniería de procesos, gracias a la cual se redefinen los procesos de la empresa en base al conocimiento obtenido en las entrevistas y se diseñan una serie de líneas de actuación que nos guían desde la situación actual hasta los objetivos definidos. Cuando las organizaciones se embarcan en la mejora del proceso, es muy importante identificar el estado actual de manera adecuada y luego establecer expectativas realistas para el estado objetivo (Dave, 2017).

En este punto nace el primer equipo de trabajo, conformado por los responsables de los departamentos y los investigadores. Este equipo es un equipo de referencia, que debe permanecer constante a lo largo de todo el estudio, y que recibe el nombre de equipo de seguimiento. Las principales funciones del equipo de seguimiento son coordinar y controlar el desarrollo del plan de actuación, así como el rendimiento de los demás equipos operativos. Es a partir de la definición de las diferentes líneas de actuación cuando se crean los equipos de trabajo más operativos, a los cuales se les asignan cada una de las líneas a implementar, pudiendo llevar cada equipo más de una línea de mejora. En estos equipos de trabajo participan, junto con los investigadores, representantes de cada uno de los departamentos, los cuáles pueden ir variando a lo largo del avance del estudio, aunque se procura mantener los equipos lo más estables posibles. Los representantes suelen ser elegidos por la propia gerencia y los responsables de cada departamento, además, el número de participantes en cada equipo es de un máximo cuatro personas sin contar a los investigadores. Cada equipo de trabajo incluye a un process leader, que es el encargado de dirigir las reuniones y facilitar el desarrollo del equipo asegurando un buen funcionamiento y evitando posibles conflictos (Whee- 
lan, 2014). En nuestro caso, esta figura es constante para todos los equipos, ya que la representa uno de los investigadores. Finalmente, se crea la figura del content leader. Este miembro de la empresa es el nexo de unión entre la compañía y los investigadores, siendo el responsable de guiar el día a día del estudio cuando los investigadores no están presentes y asistiendo a todas las reuniones de los equipos de trabajo (tanto el de seguimiento como los más operativos).

La Fase 3 se centra en la implementación y el seguimiento del plan de actuación gracias a las reuniones entre profesionales e investigadores. En los periodos en los que los investigadores no están presentes, también se llevan a cabo numerosas reuniones transversales entre los profesionales si así lo requiere la implementación de las mejoras. Estas reuniones son coordinadas por el content leader que es el encargado de coordinar y dirigir las reuniones cuando no se encuentran los investigadores en la empresa.
En cuanto al control y seguimiento del plan de actuación, se realiza a través de planes de acciones de mejora (PAM) (Figura 2). Este acta se compone de las diferentes acciones que se definen en las reuniones, a las cuales se les asigna un responsable de llevar a cabo la acción, una fecha límite y una sección de observaciones. Como es lógico, también se recoge la información sobre los asistentes a la reunión y las personas a las que irá dirigida el acta. El uso de este tipo de herramienta está orientado principalmente a reflejar los acuerdos de cada una de las reuniones a través de acciones orientadas a la consecución del objetivo final, sirviendo como método de seguimiento del avance del estudio. Así en cada una de las reuniones se comienza repasando el PAM anterior y sobre este va surgiendo el nuevo acta.

Figura 2 Plan de acciones de mejora (PAM).

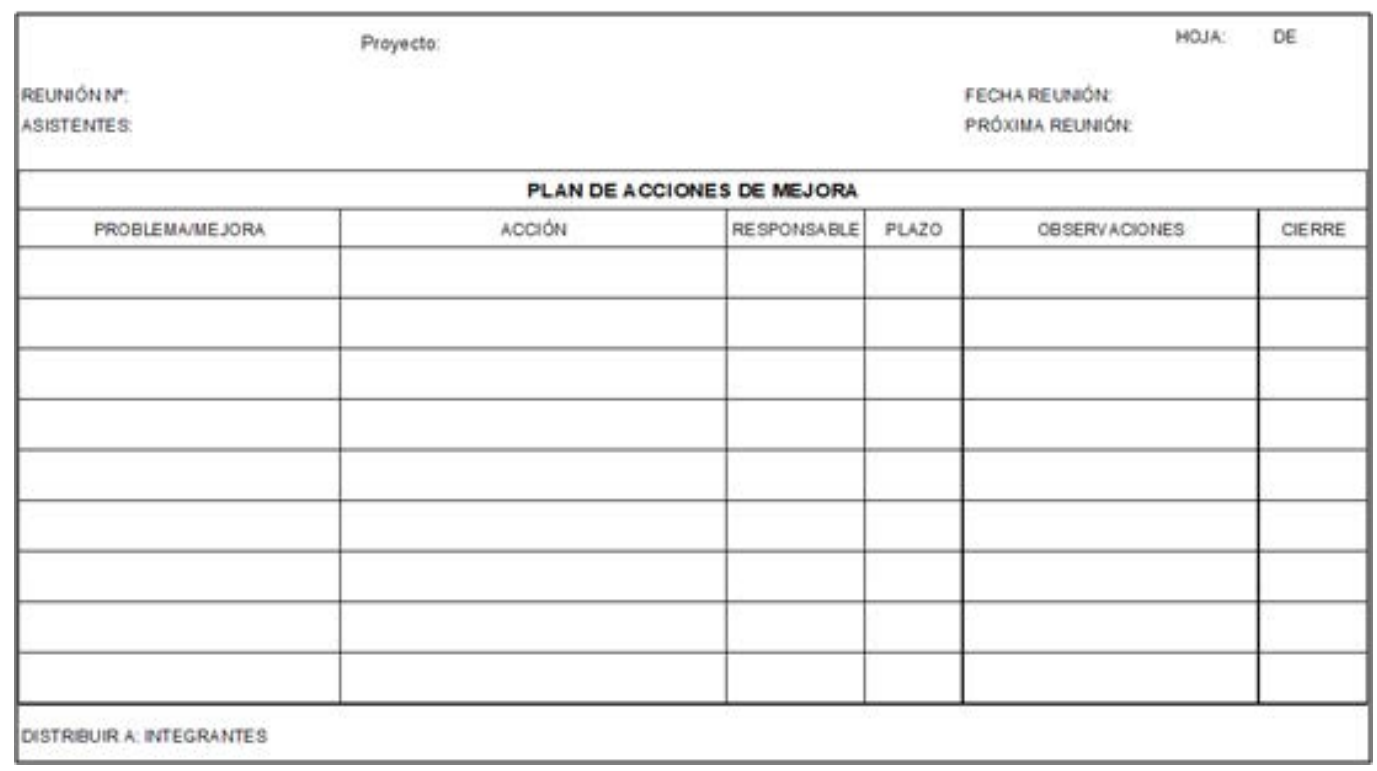

El estudio se desarrolló a lo largo del año 2017, comprendiendo dos meses la Fase 1 de conocimiento de la problemática, un mes la Fase 2 de definición de las líneas de actuación y siete meses la tercera fase de implementación y seguimiento de las mejoras. Durante las fases 1 y 2, los investigadores acudieron quincenalmente a la empresa para llevar a cabo las entrevistas con los departamentos y para definir las líneas de mejora en conjunto con todos los responsables. Durante el período de implementación, los diferentes equipos operativos se reunieron también cada 2 semanas, lo cual representó un total de 13 sesiones con cada equipo, considerando un descanso acordado con la empresa para las vacaciones de verano e invierno. Dichas sesiones estaban limitadas a 1 hora de duración. Por otro lado, el equipo de seguimiento se reunió en 4 ocasiones a lo largo de estos 7 meses (con una frecuencia aproximada de una reunión cada 2 meses), además de acudir a una sesión final extraordinaria de presentación de los resultados obtenidos.

\section{Resultados}

A partir de la metodología definida se fueron desarrollando cada una de las tres frases, desde la captación de datos hasta la implementación y seguimiento de las líneas de actuación donde se implementaron las mejoras realizadas y se cuantificaron los resultados obtenidos.

\section{Fase 1: Captación de datos}

El objetivo de la fase preparatoria fue definir la situación de la empresa a través del conocimiento interno de los profesionales y de la visión de los investigadores. Las entrevistas se llevaron a cabo con los departamentos de Comercial, Estudios y Operaciones. Principalmente, se trabajó con esos tres departamentos debido a la implicación de cada uno de ellos en la cadena de valor de la empresa. El departamento comercial es el encargado de captar clientes a los que se 
les puedan ofertar obras. Así una vez captadas las necesidades del cliente, se envía la información al departamento de Estudios, que es el encargado de realizar las ofertas. Dichas ofertas son básicamente un estudio técnico sobre las necesidades del cliente y un presupuesto de la obra que se ejecutará si se acepta la oferta. Sólo si se acepta dicha oferta, el departamento de Operaciones entra en escena. Este departamento está compuesto por jefes de obra y encargados. Los primeros gestionan más de una obra al mismo tiempo y sólo visitan las obras esporádicamente, siendo los responsables últimos de su correcta ejecución y de la rentabilidad económica de la misma. Por otro lado, los encargados son las personas responsables de ejecutar el día a día de la obra y están dedicados al cien por cien a una única obra. Además, se entrevistó a personal de los departamentos de Informática, Mejora Continua y Compras. Todas estas áreas tienen una gran implicación trasversal en todos los procesos y por ello se contó con sus aportaciones desde el inicio. Por un lado, Informática gestiona el ERP de empresa que sirve como co- lumna vertebral a todos los procesos que se llevan a cabo, Compras gestiona todos los pedidos que se realizan y finalmente el departamento de Mejora Continua lleva la gestión de la calidad y es el departamento encargado de estandarizar procesos.

Gracias a la participación de estos departamentos, y al uso de herramientas de mapeo de procesos, se fueron recogiendo las principales problemáticas e identificando las prioridades de mejora. Este análisis permitió que, dentro de cada proceso, se añadiera información complementaria sobre los principales intervinientes y las herramientas utilizadas en cada uno de ellos, logrando un mayor conocimiento. A continuación, se muestran las principales problemáticas encontradas en los equipos (Tabla 1). Los problemas detectados, además de relacionarlos con los problemas principales de la empresa, también se fueron categorizando según el departamento responsable y los departamentos a los que afectaba dicha problemática.

Tabla 1 Problemáticas definidas.

\begin{tabular}{|c|c|c|c|c|c|c|c|}
\hline \multirow{2}{*}{ № } & \multirow{2}{*}{ Problemáticas } & \multicolumn{3}{|c|}{ Departamento Responsable } & \multicolumn{3}{|c|}{ Departamento Afectado } \\
\hline & & Comercial & Estudios & Operaciones & Comercial & Estudios & Operaciones \\
\hline 1 & Problemas de captación de las necesidades del cliente & $\mathrm{x}$ & & & & $\mathrm{x}$ & $\mathrm{x}$ \\
\hline 2 & No existe un criterio para priorizar las obras a estudiar & $\mathrm{x}$ & & & & $\mathrm{x}$ & \\
\hline 3 & Ofertas mal estudiadas por falta de tiempo & $\mathrm{x}$ & & & & $x$ & \\
\hline 4 & Falta de respuestas de proveedores & & $x$ & $x$ & & $x$ & \\
\hline 5 & Procesos reiterativos en las peticiones de ofertas a proveedores & & $x$ & & & $x$ & $\mathrm{x}$ \\
\hline 6 & Falta de planificación previa al comienzo de la obra & & $\mathrm{x}$ & & & & $x$ \\
\hline 7 & Falta de control y seguimiento de la obra & & & $x$ & & & $x$ \\
\hline 8 & Rol del encargado no definido & & & $x$ & & & $x$ \\
\hline 9 & Problemas de coordinación con los subcontratistas & & & $\mathrm{x}$ & & & $x$ \\
\hline
\end{tabular}

En primer lugar, la baja conversión de ofertas en obras se relaciona con las cinco primeras deficiencias. Por un lado, el departamento Comercial presentaba la primera problemática encontrada: (1) Problemas de captación de las necesidades del cliente, lo cual afectaba enormemente a la calidad de las ofertas que posteriormente tenía que elaborar el departamento de Estudios, reduciéndose así la posibilidad de ganar la oferta. Además, cuando se enviaba información de los comerciales al departamento de Estudios sobre las obras a ofertar, nos encontrábamos con la problemática (2) No existe un criterio para priorizar las obras a estudiar, por lo que el departamento de Estudios no conocía la importancia de cada uno de los estudios que tenía que realizar. Esto era necesario para que el departamento de Estudios pudiese organizarse de la mejor manera posible, por ejemplo, poniendo más énfasis en aquellas obras que tenían más posibilidades de conseguirse. Por último, la problemática (3) Ofertas mal estudiadas por falta de tiempo, era responsabilidad del departamento de Estudios, el cual Koch (2005) ya lo consideraba como una de las fuentes de problemas más importantes en el sector de la construcción. En la mayoría de casos, los entrevistados aseguraban que esta falta de tiempo venía derivada por el tiempo dedicado a buscar proveedores. Es importante mencionar que la empresa bajo estudio no lleva a cabo el cien por cien de los trabajos en obra, sino que subcontrata muchos trabajos a proveedores especializados, por ejemplo, en instalaciones de electricidad, climatización o ventilación. En este caso, cada técnico de Estudios contactaba independientemente con los proveedores siempre que tenía que presupuestar una partida de una oferta, sin poner en común con el resto de técnicos la información sobre los servicios ofrecidos por dichos proveedores. Esta falta de información colectiva disparaba los tiempos de búsqueda.

Las líneas (4) Falta de respuestas de proveedores y (5) Procesos reiterativos en las peticiones de ofertas a proveedores, también se encuentran directamente relacionadas con esta mala gestión de los proveedores. Además de la falta de información colectiva, los mismos proveedores tenían un índice de respuesta para ofertar servicios que disminuía año tras año, habiendo proveedores que decidieron no volver a ofertar sus servicios a la empresa. Esto suponía un sobreesfuerzo para los técnicos por tener que buscar nuevos proveedores. A su vez, los investigadores se encontraron con que este problema se debía, en su origen, a que los jefes de obra (gestores de la obra una vez aceptada), decidían trabajar con sus propios proveedores de confianza y no con aquellos que habían ofertado inicialmente sus servicios. Por este motivo, numerosos proveedores dejaron de realizar presupuestos para la empresa al ver que, para muchos de los trabajos que habían ofertado, se contrataba a otros proveedores. Esta práctica no coincide con lo que Tamošaitienè et al. (2013) suponen que 
es clave en la selección de proveedores, que es el uso de modelos de decisión multicriterio que incorporen diversas características de los proveedores y que se consideran necesarios debido a la complejidad del sector de la construcción. De igual modo, diferentes autores han desarrollado otros modelos para la selección de proveedores basados en distintos criterios de análisis (Eshtehardian et al., 2013; Sarkar et al., 2017; Stević et al., 2017; Tamošaitienè et al., 2017).

En segundo lugar, la empresa necesitaba encontrar las principales razones por las cuales las obras se entregaban, en su mayoría, fuera de plazo. Las problemáticas (6) Falta de planificación previa al comienzo de la obra, (7) Falta de control y seguimiento de la obra y (8) Rol del encargado no definido, están directamente relacionadas con este hecho. Por un lado, no se realizaba correctamente, o directamente no existía, una planificación de la obra en la fase de elaboración de la oferta. Este hecho, atribuible al personal de Estudios, provocaba que se aceptasen obras inviables de realizar en el tiempo estipulado, lo cual repercutía de forma muy negativa en el rendimiento de los jefes de obra. Por norma general, en el sector de la construcción, la planificación de la producción se interpreta como la preparación de un simple diagrama de Gantt, sin preocuparse por sincronizar la información del proyecto, dejándose finalmente para la fase de ejecución de la obra (Laufer y Tucker, 1987; Henrich et al., 2005). Sin embargo, esta planificación es extremadamente importante para el éxito en cualquiera de las fases del proyecto (Zavadskas et al., 2012; Zolfani et al., 2012). Este hecho es bastante común, por eso autores como Ponz Tienda et al. (2011) realizan propuestas sobre un algoritmo de modelos cuantitativos avanzados para optimizar y controlar la secuenciación de proyectos de construcción.

Por otro lado, cabe destacar que la construcción siempre ha recibido considerable atención en cuanto a una industria que carece de un sistema de gestión estandarizado, teniendo la necesidad de mejorar los procesos (Latham 1994; Egan, 1998; Wolstenholme et al., 2009). En nuestro caso, se observó una (7) Falta de planificación y seguimiento de la obra, responsabilidad única de los jefes de obra como coordinadores de las obras. Sacks et al. (2010) destacan como factores que dificultan la coordinación las variaciones complejas en las tasas de productividad de los distintos trabajos a realizar, la falta de informes efectivos en tiempo real del progreso de las obras y la dependencia de documentos en formato papel para realizar la comunicación entre diferentes cargos. Estas mismas razones fueron expresadas por muchos de los entrevistados cuando se preguntó acerca de las deficiencias en la gestión de las obras. A mayores, el problema (8) Rol de encargado no definido, potenciaba estas deficiencias en el control, ya que los jefes de obra daban por hecho que los encargados eran los responsables de dirigir las obras a nivel operativo. Sin embargo, el perfil de encargado de obra con el que se encontraron los investigadores estaba más cercano a un operario que a un gestor, debido a que, en gran parte, el día a día de las obras impedía una reflexión adecuada sobre el pasado (lecciones aprendidas) y sobre el futuro Cárdenas y Armiñana (2009).
Estas dos problemáticas (7) y (8), también eran condicionantes evidentes del último de los problemas que asediaban a la empresa: los sobrecostes en las obras. Estos sobrecostes, ocasionados normalmente por retrabajos debidos a inexactitudes o falta de detalles, son comúnmente conocidos en el sector de la construcción (Kymmell, 2007; Eastman et al., 2011). Un incorrecto seguimiento de las obras, tanto por parte de los jefes de obra como por parte de los encargados, disparaba estos sobrecostes. Un ejemplo de nuestro caso particular eran determinados cambios solicitados por los clientes que no se registraban correctamente y que terminaban disparando los gastos adicionales. De igual modo, a raíz de las entrevistas se observaron importantes (9) Problemas de coordinación con los subcontratistas. Un ejemplo claro de esto, era el hecho de encontrarse una obra con numerosos subcontratistas sin poder trabajar debido a que otro subcontratista estaba trabajando en el mismo espacio que las primeras. Se observaba pues, que no se coordinaba el trabajo de todas ellas en conjunto, lo que en numerosas ocasiones originaba que los subcontratistas tuvieran que realizar horas extra para recuperar el tiempo perdido, disparando así los gastos de dichas partidas de trabajo y, en muchos casos, también los plazos de ejecución. Por razones como estas, es importante para las organizaciones de construcción realizar una exhaustiva integración cuando contratan empresas externas (Sarhan y Fox, 2012).

Por último, la problemática (3) Ofertas mal estudiadas por falta de tiempo, original del departamento de Estudios y que muchas veces condicionaba los ratios de conversión de ofertas en obras, se convertía en un problema aún mayor si se daba el caso de que una oferta presentada al cliente estaba incorrectamente presupuestada. Cuando una oferta presupuestada a la baja era aceptada por un cliente, era inviable ejecutarla de manera rentable por parte del departamento de Operaciones y la empresa terminaba perdiendo dinero. Como se puede observar, esta naturaleza fragmentada de la cadena de suministro, típica del sector de la construcción, da como resultado islas de información y conocimiento, tanto en relación a los proveedores como a los clientes (Alshawi e Ingirige, 2003; Aguilar y Hewage, 2013). Es por todo ello que autores como Dave et al. (2016) destacan que un flujo de información bien definido es esencial para garantizar una buena gestión y control de las obras.

\section{Fase 2: Análisis y definición de las líneas de actuación}

Una vez se tiene el conocimiento de cómo es en la actualidad el proceso y cuáles son los principales problemas del mismo, se incita a los profesionales a proponer cómo les gustaría que fuera. Esto, unido a la visión de los investigadores, conforma la definición de las principales líneas de actuación. Por tanto, una vez completado el modelado, los procesos se analizan utilizando un marco de mejora del proceso basado en los principios Lean y se van clasificando según la fase de la obra en la que se presentan. Este hecho permite ir asignando las líneas a cada uno de los equipos, definiéndose tanto acciones a corto plazo como a largo plazo. A continuación, 
en la Figura 3 se muestra un esquema donde a partir de las problemáticas definidas en la Fase 1, se detallan las líneas de actuación y se asignan a los diferentes equipos de trabajo que abordarán cada una de ellas.
Figura 3 Relación entre las problemáticas encontradas y las líneas de actuación asignadas a los equipos de trabajo.

\begin{tabular}{|c|c|c|}
\hline Problemáticas & № & Equipos de trabajo \\
\hline Problemas de captación de las necesidades del cliente & 1 & \multirow{3}{*}{$\begin{array}{l}\text { Estudios-Comercial } \\
\text { 1. Definición de un checklist para } \\
\text { captar la información de los clientes } \\
\text { 2. Creación de un panel Kanban } \\
\text { 3. Estandarización de las ofertas }\end{array}$} \\
\hline No existe un criterio para priorizar las obras a estudiar & 2 & \\
\hline Ofertas mal estudiadas por falta de tiempo & 3 & \\
\hline Falta de respuestas de proveedores & 4 & \multirow{2}{*}{$\begin{array}{l}\text { Estudios-Compras-Operaciones } \\
\text { 4. Creación de una base de datos de } \\
\text { proveedores } \\
\text { 5. Mejora de la coordinación y } \\
\text { comunicación interna de la obra }\end{array}$} \\
\hline Procesos reiterativos en las peticiones de ofertas a proveedores & 5 & \\
\hline Falta de planificación previa al comienzo de la obra & 6 & \multirow{3}{*}{$\begin{array}{l}\text { Operaciones } \\
\text { 6. Definición de procedimientos } \\
\text { estándares para la gestión de la obra } \\
\text { 7. Jornada de formación para los } \\
\text { encargados }\end{array}$} \\
\hline Alta dedicación de los técnicos en la búsqueda de proveedores & 7 & \\
\hline Falta de control y seguimiento de la obra & 8 & \\
\hline Problemas de coordinación con los subcontratistas & 9 & \\
\hline
\end{tabular}

A través de la creación de los tres equipos de trabajo se perseguía el objetivo de abordar las diferentes líneas de actuación. Así se definió el plan de actuación y se presentó a los diferentes equipos para ir asignando las responsabilidades a los diferentes miembros de cada uno de los equipos. Por un lado, se trabajó con el equipo de trabajo de Operaciones que estaba formado únicamente por los diferentes jefes de obra. Por otro lado, se crearon dos equipos mixtos, uno de ellos formado por los departamentos de Estudios y Comercial, mientras el otro equipo estaba compuesto por representantes de los departamentos de Estudios, Compras y Operaciones. A pesar de no haber participado en la primera fase, por la importancia de algunas líneas como es la creación de una base de datos común, en este último equipo de trabajo se invitó a participar al personal de Compras. Las siete líneas de actuación principales se describen a continuación. Para ayudar en la comprensión de cada acción, se presentan por separado y en relación a cada equipo de trabajo.

El equipo de trabajo formado por representantes del departamento de Estudios y Comercial trabajó principalmente con tres líneas:

1. Definición de un checklist para captar la información de los clientes: esta mejora estuvo directamente orientada a mejorar la captación de necesidades de los clientes. El checklist que se diseñó, no contenía sólo información comercial, sino que incluyó apartados técnicos básicos que eran muy relevantes para el departamento de Estudios; todo ello procurando que la recogida de esta información no supusiese un esfuerzo extra para los comerciales.

2. Creación de un panel Kanban: este tipo de herramientas son bien conocidas y se usan regularmente en la metodología Lean ya que ayudan a hacer la comunicación simple, atractiva y eficiente. Este método forma parte de una técnica de gestión llamada Visual Management, cuya aplicación está aumentando en la construcción (Tezel, 2011). En nuestro caso, la herramienta Kanban estuvo orientada a mejorar la coordinación entre los departamentos de Comercial y Estudios. Las tarjetas Kanban eran generadas por el departamento Comercial e incluyeron toda la información relevante de una obra a estudiar: cliente, plazo de entrega y requisitos especiales de la oferta a elaborar. Además, en dicha tarjeta el departamento Comercial indicaba el porcentaje aproximado sobre la probabilidad de ganar la obra y a la vez asignaba un valor entre 1 y 4 a la obra en función de su prioridad (de menos a más prioritaria). Seguidamente, las tarjetas eran recibidas por el director del departamento de Estudios, que asignaba la obra a un técnico para que comenzara a estudiarla y a continuación la colgase en el panel Kanban. Este panel estaba compuesto por diversas filas (una por cada técnico de Estudios) y columnas (estado de avance de la oferta). Esta disposición ayudaba al departamento de Estudios a priorizar y nivelar el trabajo de mejor manera en su equipo. Una vez la oferta se terminaba, los propios técnicos de Estudios actualizaban la tarjeta Kanban con información importante, como bien podía ser el importe de la oferta presentada al cliente o la fecha real de finalización de la oferta. A continuación, se movía la tarjeta a la columna final de 'Esperando Respuesta' para que finalmente las tarjetas se retirasen del panel una vez las ofertas se rechazaban o aceptaban.

3. Estandarización de las ofertas: en este caso, se buscaba que todos los técnicos trabajasen de la misma forma, reducir tiempos innecesarios en labores que no añadían valor y centrar esfuerzos en las labores más importantes. A este respecto, se buscaba estandarizar el tipo de oferta en función de la prioridad que el departamento Comercial hubiera asignado a la obra, dedicándole más esfuerzo a aquellas de mayor prioridad y terminando así de alinear el trabajo entre ambos departamentos. Algunos de los elementos que se pretendían estandarizar eran: la incorporación a la oferta de un planning aproximado de la ejecución de la obra, el número de ofertas que se pedían a proveedores por cada partida a estudiar y el importe máximo hasta el cual ciertas partidas se podían presupuestar en base a históricos, es decir, sin pedir oferta a nuevos proveedores. 
El segundo equipo mixto formado por los departamentos de Estudios, Compras y Operaciones se centró en dos grandes líneas de trabajo:

4. Creación de una base de datos de proveedores: los departamentos de Estudios y de Operaciones pusieron en común los proveedores más utilizados, creando un pool de proveedores en el ERP de la empresa al que todos los departamentos tuvieran acceso. Además, se programaron reuniones iniciales entre los integrantes de Estudios, Compras y Operaciones para poner en común cuestiones cualitativas acerca de los proveedores más importantes, con el objetivo de alinear ideas y priorizar la selección de unos u otros proveedores. En el ERP se introdujeron numerosas características de los proveedores, como eran el área geográfica donde operaban o la naturaleza de los servicios prestados, facilitando enormemente la búsqueda de los mismos por parte de los integrantes de Estudios. Además, para conseguir ampliar y dar estabilidad a la cartera de proveedores se crearon dos acuerdos. Por un lado, un acuerdo con el departamento de Estudios para que, además de pedir ofertas a los proveedores de la base de datos para cada una de las partidas de un presupuesto, también se solicitase, como mínimo, una oferta por partida a un proveedor nuevo y así comparar precios. Por otro lado, un acuerdo con el departamento de Operaciones para que los jefes de obra siempre escogiesen a uno de los proveedores analizados por el departamento de Estudios, evitando que los proveedores presupuestaran continuamente sin recibir ninguna obra.

5. Mejora de la coordinación y comunicación interna de la obra: esta línea de actuación se basó en fomentar la colaboración entre los diferentes departamentos una vez que una oferta era aceptada por parte de un cliente y se convertía en una obra a ejecutar. En primer lugar, con la obra ya adjudicada, el departamento de Estudios debía enviar la oferta aceptada a los departamentos de Operaciones y Compras para que analizasen la obra. Además, se decidió centralizar en el ERP la información de las ofertas para facilitar el seguimiento por parte de cualquier otro departamento. Tras un período menor a una semana, el departamento de Estudios tenía la responsabilidad de convocar una reunión a la que acudiesen el comercial que contactó con el cliente, el técnico de Estudios que hubiese estudiado la obra, el comprador asignado, el jefe de obra que gestionaría la obra y el encargado de la obra. Dicha reunión tenía como objetivo principal discutir la viabilidad del planning inicial y del presupuesto elaborado por Estudios, para analizar posibles alternativas en caso de que el jefe de obra considerase que no era posible llevarlo a cabo. Además, la estacionalidad ocasionada por la subcontratación de los trabajos en obra delimitaba la flexibilidad y eficiencia en el control de los procesos, razón por la cual estas reuniones también servían para planificar las entradas y salidas de los subcontratistas (proveedores), dándole mayor peso a aquellas partidas críticas por plazos o por importe (Urueña, 2011). Por último, se trataba también todo lo relativo a necesidades de compra y alquiler de materiales y maquinaria, de lo cual se encargaba el departamento de Compras.

El equipo formado por el departamento de Operaciones se centró en dos líneas principales:

6. Definición de procedimientos estándares para la gestión de la obra: está línea de mejora se trabajó en exclusiva con el departamento de Operaciones con el objetivo de definir procesos y procedimientos para mejorar la gestión de una obra. Por un lado, se creó numerosa documentación necesaria para estandarizar el control de la obra, entre las que se pueden destacar: registros de incidencias, tanto propias como de clientes y proveedores, actas de reuniones con clientes, Planes de Puntos de Inspección (PPIs) para los controles de calidad $\mathrm{y}$, finalmente, plantillas de seguimiento de evolución de la obra, las cuales servían para medir el avance de cada una de las partidas ejecutadas por los distintos subcontratistas. Además, se estandarizó el uso del ERP para el seguimiento económico de la obra, pasando a introducirse todos los costes de las obras semanalmente (antes se hacía de manera mensual), lo cual nos permitió detectar desviaciones en los gastos con mayor rapidez.

En lo relativo a la coordinación con otros interesados (tanto internos como externos), se definieron tres tipos de reuniones que ayudaron a mejorar el grado de conocimiento de los mismos sobre el avance de la obra. En primer lugar, para el lanzamiento de la obra se establecieron las conocidas como Pull Sesions. Estas reuniones fueron diseñadas para que el jefe de obra y el encargado de la obra, conjuntamente con los subcontratistas más relevantes, analizasen la planificación antes de empezar la obra. Se llevó a cabo, por tanto, una replanificación conjunta a medio plazo de los trabajos de los subcontratistas, con el objetivo de encontrar sinergias y eliminar posibles restricciones. Este modelo de Pull Sesion está bastante ligado con lo que en la literatura se identifica como Last Planner System, que persigue medir las desviaciones de la planificación inicial y asociar los motivos de la falta de rendimiento para conocer la causa raíz de los problemas (Ballard, 2000). En segundo y tercer lugar, con una frecuencia semanal, se establecieron reuniones tanto de coordinación de la obra como de seguimiento con el cliente. En las reuniones de coordinación de la obra, el encargado debe adquirir el rol de líder y programar el trabajo del día de manera coordinada con los encargados de los subcontratistas, lo que se traducía en una especie de Pull Sesions más operativas. Para las reuniones de seguimiento con el cliente, se consideró necesario la presencia del jefe de obra, responsable último del éxito de la misma. Cabe señalar que, en caso de obras de gran complejidad y/o volumen, estos dos tipos de reuniones se realizarían diariamente. Se destaca, por tanto, la importancia de la participación de los profesionales que llevarán a cabo el trabajo de ejecución de la obra desde las fases más tempranas del proyecto, enmarcado en un proceso 
de diseño colaborativo con metas de coste y plazo comunes (Harvey, 2003, Cárdenas y Armiñana, 2009; Herranz Limon, 2015).

7. Jornada de formación para los encargados: en línea con todos los cambios que se expusieron en el apartado 6, es evidente que la figura del encargado de obra adquiere un rol que hasta la fecha no tenía. Debido a las nuevas funciones de gestión atribuibles a los encargados, se consideró importante diseñar e impartir una formación específica que ayudara a inculcar estos cambios. La formación se centró en la necesidad de organizar y gestionar recursos materiales y humanos en la obra, y en reforzar las labores de supervisión y control que requiere el puesto. Paralelamente la formación buscó mostrar la importancia del liderazgo del encargado, la comunión efectiva y la sensibilización de los trabajadores de los subcontratistas en los procesos de ejecución de la misma, estableciendo al cliente como punto de encuentro de cualquier desacuerdo posible. La formación se organizó en una sesión única de 4 horas a la que acudieron todos los encargados, los jefes de obra y otros implicados en el proceso de ejecución de la obra. Para su desarrollo se utilizó el método del caso (una historia real sobre el día a día de una obra) para potenciar el impacto de la formación en el personal y fueron los propios investigadores quienes desarrollaron dicha formación.

\section{Fase 3: Implementación y seguimiento del plan de ac- tuación}

Después de definir el plan de actuación, el estudio pasó a la última fase: implementación y seguimiento del plan de actuación. En esta sección se presenta una reflexión sobre los resultados de cada acción, incluyendo también aquellos resultados inesperados, y se da una visión sobre la evolución del estudio. Como se observa en la Tabla 2, de las siete líneas de actuación planteadas y definidas, cuatro de ellas se acometieron con éxito, dos sólo se alcanzaron parcialmente y la restante se descartó ya con el estudio en curso. Como es lógico, dicho grado de implementación dependió del comportamiento de los equipos. La clave en este tipo de estudios es que los profesionales asuman un papel muy activo tanto en la definición de la problemática como en la implementación de las acciones (Rydenfält et al., 2017).
Tabla 2 Grado de implementación de las diferentes líneas definidas.

\begin{tabular}{|l|l|l|l|l|}
\hline & \multicolumn{1}{|c|}{ Líneas de actuación } & Descartada & $\begin{array}{c}\text { Parcialmente } \\
\text { completada }\end{array}$ & Completada \\
\hline 1 & Definición de un checklist para captar la información de los clientes & & & \\
\hline 2 & Creación de un panel Kanban & & & \\
\hline 3 & Estandarización de las ofertas & & & \\
\hline 4 & Creación de una base de datos de proveedores & & & \\
\hline 5 & Mejora de la coordinación y comunicación interna de la obra & & & \\
\hline 6 & Definición de procedimientos estándares para la gestión de la obra & & & \\
\hline 7 & Jornada de formación para los encargados & & & \\
\hline
\end{tabular}

cuando a partir del cuarto mes, el departamento Comercial comenzó a asignar una preferencia máxima a prácticamente todas las obras que tenía que presupuestar el departamento de Estudios. Esto ocasionó que, de nuevo, fuera muy complicado gestionar la carga de trabajo en el departamento de Estudios, que se vio sobresaturado. Esta excesiva carga de trabajo terminó por complicar todavía más la situación en el departamento, ya que se produjeron numerosas salidas de personal técnico, ocasionando desequilibrios importantes en el equipo de mejora. Debido a estas complicaciones, a lo largo del estudio se intentó que el equipo de seguimiento, el cual incorporaba a todos los responsables de los departamentos y a la gerencia, marcara las pautas a seguir. Sin embargo, las diferencias entre los departamentos de Comercial y Estudios llegaba hasta los niveles jerárquicos más altos, haciendo imposible buscar un consenso. Por último, la estandarización de las ofertas elaboradas por el departamento de Estudios sí que se logró, aunque no fue hasta el último mes del estudio cuando se definió el estándar definitivo. Esto fue, en gran medida, debido a la alta rotación del departamento mencionada anteriormente, lo que ocasionó que las visiones de los integrantes del equipo fueran cambiando constantemente dificultando el llegar a un acuerdo. panel, que debería servir como herramienta de coordinación entre ambos departamentos, terminó por perder su utilidad 


\section{Equipo formado por los departamentos de Estudios, Compras y Operaciones}

Completaron con éxito tanto la mejora (4) Creación de una base de datos de proveedores, como la (5) Mejora de la coordinación y comunicación interna de la obra, siendo el equipo que mejor rendimiento tuvo. Respecto a la primera de ellas, fue el departamento de Compras el que acogió el liderazgo de esta línea de actuación y fomentó la colaboración continua de los integrantes de los departamentos de Estudios y Operaciones. Tras los siete meses de implementación, más de 300 proveedores estaban dados de alta en la base de datos. A este respecto, consideramos que el éxito de esta mejora se debe a que la de la base de datos se empezó a construir con la información de los proveedores de las zonas donde más obras se llevaban a cabo. Gracias a ello, los técnicos de Estudios que presupuestaban las obras pudieron hacer uso de la base de datos al poco tiempo de su lanzamiento. Estos pequeños logros motivaron al equipo $\mathrm{y}$, desde nuestro punto de vista, supusieron el éxito al final del estudio. En cuanto a la mejora de la coordinación y comunicación interna de la obra, fue algo inherente al estudio. Las mismas reuniones que llevábamos a cabo con el equipo servían para poner encima de la mesa numerosos problemas de coordinación, los cuales se trataban en esas reuniones, proponiendo soluciones in situ. En esta línea de actuación en particular, el rol del content leader fue de enorme importancia, ya que era la persona encargada de hacer un seguimiento de todas estas pequeñas mejoras ligadas al día a día de la empresa. Además, los técnicos de Estudios mostraron un nivel de implicación extra para mejorar en este aspecto, adaptando muchos de sus procesos (incluyendo el modelo estándar de oferta descrito anteriormente) a las necesidades de los compradores y los jefes de obra, lo cual brindó enormes mejoras al rendimiento de los tres departamentos.

\section{Equipo formado por el personal de Operaciones}

Este último equipo, integrado por los jefes de obra, logró grandes avances en las líneas de mejora que le correspodían. Sin embargo, cabe destacar la enorme dificultad presentada para llevar a cabo las reuniones periódicas con este equipo, y en consecuencia el seguimiento del estudio. Este hecho se debió a la enorme movilidad geográfica de sus integrantes, los cuales viajaban constantemente a las distintas obras que estaba ejecutando la empresa. Es por ello que la mejora (6) Definición de procedimientos estándares para la gestión de la obra, la consideramos como una mejora parcialmente alcanzada. En primer lugar, toda la documentación necesaria para el seguimiento estándar de la obra se generó y aprobó en consenso con los propios jefes de obra. Además, se estandarizó el uso del ERP siendo uno de los propios jefes de obra el que formó a los demás para alinear la forma de trabajar. Finalmente, todas las reuniones de coordinación con subcontratistas y clientes se comenzaron a llevar a cabo, convirtiéndose finalmente en un estándar. El único punto pendiente fue, en referencia a toda la documentación que se creó para obtener un mayor control sobre las obras, el uso riguroso y sistemático de dicha documentación. La misma movilidad de los jefes de obra que condicionó las reuniones del equipo de mejora, también condicionó el control por parte del content leader sobre la aplicación o no de las mejoras implantadas. En concreto, este punto fue crítico a lo largo del estudio, habiendo disparidad entre el compromiso de los distintos jefes de obra sobre llevar el control que se les requería. Finalmente, la mejora (7) Jornada de formación para los encargados, se realizó con éxito y fue avalada tanto por los encargados como por los mismos jefes de obra, los cuales observaron como gran parte del trabajo que realizaban podía ser delegado en sus trabajadores. Esta formación fue la que sirvió para extender las mejoras definidas y trabajadas en el equipo de los jefes de obra a la realidad del día a día de las obras que viven los encargados.

En conjunto, estas siete líneas de mejora independientes y trabajadas en paralelo nos acercaron a la consecución de los objetivos principales del estudio. Por un lado, la conversión de ofertas en obras se vio directamente implicada gracias a la creación de las ofertas estándar y a la base de datos de proveedores, que simplificaron el trabajo del departamento de Estudios y facilitaron la elaboración de ofertas de mayor calidad. Tampoco se puede obviar que la mejora en la captación de las necesidades del cliente hubiese generado un cambio aún mayor en este sentido, y sin embargo no se llevó a cabo mejora alguna a ese respecto. Las pruebas sin éxito también deben documentarse, no solo por razones de objetividad, sino porque pueden ayudar a los profesionales a seleccionar modelos de diseño e identificar posibles problemas de implementación (van Aken et al., 2016). Por otro lado, tanto los sobrecostes en las obras como los retrasos se redujeron de forma considerable gracias a la mejora en la coordinación de todos los implicados en el estudio y la ejecución de la obra, desde los departamentos propios de la empresa hasta los subcontratistas.

\section{Conclusiones}

El uso de la metodología Action Research, inspirada en el aprendizaje activo, facilita el desarrollo organizacional y la capacidad de mejora, mientras que al mismo tiempo se tratan los desafíos más complejos de la industria de la construcción. En este estudio, se propuso una metodología para facilitar el desarrollo organizacional basándose en el uso de herramientas de Lean Construction. En general, las mejoras implementadas en este estudio se pueden considerar un logro a pesar del distinto grado de cumplimiento de las líneas de actuación planteadas. Los equipos lograron afrontar con éxito muchas problemáticas, alcanzando los tres objetivos iniciales que se plantearon: mejorar el ratio de obtención de proyectos respecto al número de ofertas elaboradas y reducir los sobrecostes y las obras entregadas fuera de plazo. 
Se considera que la metodología seguida funcionó correctamente a nivel grupal, ya que los diferentes equipos se centraron en tareas muy específicas y se establecieron niveles de confianza suficientes entre los departamentos para permitir diálogos abiertos en el desarrollo de las reuniones. El seguimiento de las líneas de actuación a través de los planes de acciones de mejora (PAMs) permitió un control sistemático del avance del estudio, además de servir como herramienta para afianzar el compromiso de los implicados con los equipos de mejora, haciendo responsable a cada individuo de las diferentes acciones que se llevaron a cabo. Otra herramienta de seguimiento importante hubieran sido las reuniones con el equipo de seguimiento (compuesto por los responsables de los distintos departamentos y la gerencia), cuyo fin era redirigir cada una de las líneas de actuación en los momentos más críticos. Sin embargo, el no haber conseguido que el departamento Comercial y el de Estudios solucionasen sus discrepancias y condicionaran las mejoras de ese equipo, lo consideramos un fracaso por parte de este equipo directivo. Al contrario, el rol del content leader sí que fue vital en el desarrollo del estudio ya que las reuniones informales con los investigadores ayudaron a aclarar muchas contrariedades del estudio que sólo se observan en el día a día de la empresa y que los investigadores por su cuenta no serían capaces de captar. Además, por la parte de los investigadores, creemos que la aplicación de nuestra metodología de Action Research logró transmitir a la organización nuestra firme intención de hacer, más que de observar y registrar, y así llegar a un resultado más útil que el del investigador como un simple observador. Finalmente, cabe destacar que, gracias a esta metodología, la identificación e implementación de oportunidades de mejora, además de ayudar a los resultados de la empresa, genera y consolida conocimiento en cada uno de los integrantes de los equipos de trabajo.

En lo relativo a la literatura en el ámbito de la construcción, y tras analizar la situación de la empresa y compararla con la literatura relativa a las principales problemáticas presentes en el sector, se han encontrado diferencias significativas. A lo largo de los años, la literatura en Lean Construction se ha centrado, principalmente, en los problemas originados durante el transcurso de las obras. Sin embargo, este estudio muestra como los problemas de este sector nacen, en gran parte, de problemas transversales a los diferentes departamentos que gestionan no sólo la obra, sino también todos los procesos previos a la ejecución de la misma.

Por último, aunque la metodología seguida nos ha permitido obtener resultados notables, somos conscientes de que nuestro estudio tiene algunas limitaciones. Por un lado, no se llega al contacto directo con el personal de obra. Este hecho, unido a la falta de participación de los jefes de obra por alta carga de trabajo, limita el alcance del estudio en el ámbito de la ejecución de las obras. Futuros estudios podrían centrarse en la aplicación de herramientas de Lean Construction que conecten todavía más los procesos de gestión y de ejecución de las obras. Por otro lado, no se ha llevado a cabo una obra piloto en la que se pusieran en práctica las mejoras aplicadas y se comparara con una obra gestionada antes de la implementación. De esta forma se podría analizar los resultados de una manera más cuantitativa. La técnica de las implantaciones piloto en entornos industriales es muy común para poder ir cuantificando las mejoras (Dave et al., 2016). Sin embargo, la falta de homogeneidad en los tipos de obras que se ejecutaban hizo muy difícil la comparativa. En un futuro, se podrían desarrollar modelos comparativos que facilitasen este análisis y diesen un mayor valor a las mejoras logradas. Además, como última línea futura de investigación, creemos que sería interesante aplicar la metodología de Action Research aquí presentada en otros desafíos dentro del mismo sector o, incluso, en otros entornos donde profesionales multidisciplinares trabajen en equipo para resolver problemáticas trasversales a las organizaciones. El conocimiento ganado puede servir para reestructurar las normas de la organización y al mismo tiempo aportar información valiosa para la comunidad científica para futuras investigaciones.

\section{Referencias}

1. Aguilar, G. E., \& Hewage, K. N. (2013). IT based system for construction safety management and monitoring: C-RTICS 2. Automation in Construction, 35, 217228.

2. Alshawi, M., \& Ingirige, B. (2003). Web-enabled project management: an emerging paradigm in construction. Automation in construction, 12(4), 349-364.

3. Azambuja, M., \& O’Brien, W. J. (2009). Construction supply chain modeling: issues and perspectives. Construction supply chain management handbook, 1 .

4. Ballard, H. G. (2000). The last planner system of production control (Doctoral dissertation, University of Birmingham).

5. Berner, F., Hermes, M., Lange, S., \& Graeser, D. (2016). Lean Construction in the logistics of underground and road construction Optimization of construction in constricted conditions. BAUINGENIEUR, 91, 166-171.

6. Brydon-Miller, M., Greenwood, D., \& Maguire, P. (2003). Why Action Research?.

7. Cárdenas, L. F. A., \& Armiñana, E. P. (2009). Un nuevo enfoque en la gestión: la construcción sin pérdidas. Revista de Obras Públicas, 46. 
8. Choma, J., Zaina, L. A., \& Da Silva, T. S. (2015, September). Towards an Approach Matching CMD and DSR to Improve the Academia-Industry Software Development Partnership: A Case of Agile and UX Integration. In Software Engineering (SBES), 2015 29th Brazilian Symposium on (pp. 51-60). IEEE.

9. Coughlan, P., \& Coghlan, D. (2002). Action research for operations management. International journal of operations \& production management, 22(2), 220-240.

10. Dave, B. (2017). Business process management-a construction case study. Construction Innovation, 17(1), 5067.

11. Dave, B., Kubler, S., Främling, K., \& Koskela, L. (2016). Opportunities for enhanced Lean Construction management using Internet of Things standards. Automation in Construction, 61, 86-97.

12. Eastman, C. M., Eastman, C., Teicholz, P., \& Sacks, R. (2011). BIM handbook: A guide to building information modeling for owners, managers, designers, engineers and contractors. John Wiley \& Sons.

13. Egan, J. (1998). Rethinking construction, construction task force report for department of the environment, transport and the regions. ed: HMSO, London.

14. Eshtehardian, E., Ghodousi, P., \& Bejanpour, A. (2013). Using ANP and AHP for the supplier selection in the construction and civil engineering companies; case study of Iranian company. KSCE Journal of Civil Engineering, 17(2), 262-270.

Fagundes, L. D., Amorim, E. S., \& da Silva Lima, R. (2017). Action research in reverse logistics for end-of-life tire recycling. Systemic Practice and Action Research, 30(5), 553-568.

15. Farooq, S., \& O'Brien, C. (2015). An Action Research methodology for manufacturing technology selection: a supply chain perspective. Production Planning \& Control, 26(6), 467-488.

16. Gutierrez, D. M., Scavarda, L. F., Fiorencio, L., \& Martins, R. A. (2015). Evolution of the performance measurement system in the Logistics Department of a broadcasting company: An Action Research. International Journal of Production Economics, 160, 1-12.
17. Haj-Bolouri, A., Bernhardsson, L., \& Rossi, M. (2016). PADRE: A Method for Participatory Action Design Research. In Tackling Society's Grand Challenges with Design Science: 11th International Conference, DESRIST 2016, St. John's, NL, Canada, May 23-25, 2016, Proceedings 11 (pp. 19-36). Springer International Publishing.

18. Harvey, M. (2003). Privatization, fragmentation and inflexible flexibilization in the UK construction industry. Building chaos: An international comparison of deregulation in the construction industry, 188-209.

19. Henrich, G., Tilley, P., \& Koskela, L. (2005). Context of production control in construction.

20. Herranz Limon, D. (2015). Measuring Lean Construction-A Performance Measurement model supporting the implementation of Lean practices in the Norwegian construction industry (Master's thesis, NTNU).

21. Hjalmarsson, A., Rudmark, D., \& Lind, M. (2010). When designers are not in control-experiences from using Action Research to improve researcher-developer collaboration in design science research. Global perspectives on design science research, 1-15.

22. Kagioglou, M., Cooper, R., Aouad, G., \& Sexton, M. (2000). Rethinking construction: the generic design and construction process protocol. Engineering, Construction and Architectural Management, 7(2), 141-153.

23. Kaipia, R., Holmström, J., Småros, J., \& Rajala, R. (2017). Information sharing for sales and operations planning: Contextualized solutions and mechanisms. Journal of Operations Management.

24. Kymmell, W. (2007). Building Information Modeling: Planning and Managing Construction Projects with 4D $\mathrm{CAD}$ and Simulations (McGraw-Hill Construction).

25. Koch, C. (2005). Failures in combined knowledge and material supply chains. In International Engineering Management Conference. IEEE.

26. Kolb, D. A. (1984). Experience as the source of learning and development Prentice-Hall. Englewood Cliffs.

27. Koskela, Lauri (1992). Application of the New Production Philosophy to Construction. Center for Integrated Facility Engineering, Stanford University, USA. 
28. Kumaraswamy, m. m. (1999). Profiting from harmony-in construction procurement and management. Profitable Partnering in Construction Procurement, 85-92.

29. Latham, M. (1994). Constructing the team: final report: joint review of procurement and contractual arrangements in the United Kingdom construction industry. HMSO Books.

30. Laufer, A., \& Tucker, R. L. (1987). Is construction project planning really doing its job? A critical examination of focus, role and process. Construction Management and Economics, 5(3), 243-266.

31. McGrath, R. G. (2001). Exploratory learning, innovative capacity, and managerial oversight. Academy of management journal, 44(1), 118-131.

32. McManners, P. (2016). The Action Research case study approach: A methodology for complex challenges such as sustainability in aviation. Action ResearchAction Research, 14(2), 201-216.

33. Paim, R., Mansur Caulliraux, H., \& Cardoso, R. (2008). Process management tasks: a conceptual and practical view. Business Process Management Journal, 14(5), 694-723.

34. Ponz Tienda, J. L., Benlloch Marco, J., Andrés Romano, C., \& SENABRE, D. (2011). Un algoritmo matricial RUPSP/GRUPSP" sin interrupción" para la planificación de la producción bajo metodología Lean Construction basado en procesos productivos. Revista de la Construcción, 10(2), 90-103.

35. Rydenfält, C., Larsson, P. A., \& Odenrick, P. (2017). An action-oriented method for interprofessional organization development at a hospital operating unit. Action ResearchAction Research, 15(2), 177-197.

36. Sacks, R., Radosavljevic, M., \& Barak, R. (2010). Requirements for building information modeling based Lean production management systems for construction. Automation in construction, 19(5), 641-655.

37. Sarhan, S., \& Fox, A. (2012). Trends and challenges to the development of a Lean culture among UK construction organisations'. In Proceedings for the 20th Annual Conference of the IGLC (pp. 1151-1160).

38. Sarkar, S., Lakha, V., Ansari, I., \& Maiti, J. (2017). Supplier Selection in Uncertain Environment: A Fuzzy MCDM Approach. In Proceedings of the First International Conference on Intelligent Computing and Communication (pp. 257-266). Springer Singapore.
39. Stević, Ž., Pamučar, D., Vasiljević, M., Stojić, G., \& Korica, S. (2017). Novel Integrated Multi-Criteria Model for Supplier Selection: Case Study Construction Company. Symmetry, 9(11), 279.

40. Sunding, L., \& Odenrick, P. (2010). A method for Action Research interventions to improve joint problem solving in operational teams in the Swedish construction industry. Qualitative Research in Accounting \& Management, 7(1), 97-123.

41. Tamošaitienè, J., Zavadskas, E. K., \& Turskis, Z. (2013). Multi-criteria risk assessment of a construction project. Procedia Computer Science, 17, 129-133.

42. Tamošaitienè, J., Zavadskas, E. K., Šileikaitė, I., \& Turskis, Z. (2017). A novel hybrid MCDM approach for complicated supply chain management problems in construction. Procedia Engineering, 172, 1137-1145.

43. Teicholz, P., Goodrum, P. M., \& Haas, C. T. (2001). US construction labor productivity trends, 1970-1998. Journal of Construction Engineering and Management, 127(5), 427-429.

44. Tezel, B. A. (2011). Visual management: an exploration of the concept and its implementation in construction (Doctoral dissertation, Salford: University of Salford).

45. Tezel, A., Koskela, L., \& Aziz, Z. (2017). Current condition and future directions for Lean Construction in highways projects: a small and medium-sized enterprises (SMEs) perspective. International Journal of Project Management.

46. Urueña, J. F. C. (2011). Modelos de Integración de la Gestión de la Cadena de Suministro en el Sector de la Construcción. Dirección y Organización, (43), 51-63.

47. van Aken, J., Chandrasekaran, A., \& Halman, J. (2016). Conducting and publishing design science research: Inaugural essay of the design science department of the Journal of Operations Management. Journal of Operations Management, 47, 1-8.

48. Wheelan, S. A. (2014). Creating effective teams: A guide for members and leaders. Sage Publications.

49. Wolstenholme, A., Austin, S. A., Bairstow, M., Blumenthal, A., Lorimer, J., McGuckin, S., ... \& Guthrie, W. (2009). Never waste a good crisis: a review of progress since Rethinking Construction and thoughts for our future. 
50. Yin, R. K. (2014). Case study research: Design and methods.

51. Zavadskas, E. K., Vainiūnas, P., Turskis, Z., \& Tamošaitiené, J. (2012). Multiple criteria decision support system for assessment of projects managers in construction. International Journal of Information Technology \& Decision Making, 11(02), 501-520.
52. Zolfani, S. H., Chen, I. S., Rezaeiniya, N., \& Tamošaitienè, J. (2012). A hybrid MCDM model encompassing AHP and COPRAS-G methods for selecting company supplier in Iran. Technological and economic development of economy, 18(3), 529-543. 